\title{
PROJECTIONS OF ZERO-SETS (AND THE FINE UNIFORMITY ON A PRODUCT)
}

\author{
BY \\ ANTHONY W. HAGER
}

1. Introduction. We present here some results asserting that, under certain conditions on the pair of topological spaces $(X, Y)$, the projection $\pi_{X}$ of $X \times Y$ onto $X$ is " $z$-closed", i.e., carries zero-sets onto closed sets. These results are intended to contribute to the description of the fine uniformity on a product space, via the following. (Proof in $\S 6$, see also [N, 1.6].)

1.1. The semi-uniform product $X * Y$ of fine uniform spaces $X$ and $Y$ is fine iff $\pi_{X}$ is $z$-closed.

(The terminology on uniform spaces follows $\left[I_{1}\right]$. We consider only completely regular Hausdorff spaces. A zero-set is the set of zeros of a real-valued continuous function.)

For comparison with our results, we state the following theorem, due to Isbell, using results and methods of Glicksberg, Frolík, and Onuchic.

$1.2\left[\mathrm{I}_{1}\right.$, Chapter VII]. The uniform product of two fine uniform spaces is fine iff either (a) for some cardinal $n$, one factor is discrete of power $n$, and the other is $n$-discrete, or, (b) for some cardinal $n$, the product is pseudo-n-compact and m-discrete for all $m<n$.

(Some of these terms are defined below.)

Since the uniformity of the semi-uniform product is finer (larger) than that of the uniform product, each set of conditions in 1.2 is sufficient that $\pi_{X}$ be $z$-closed. (In fact, see [N, 1.7].)

We point out explicitly that we have not obtained a complete classification of circumstances under which $\pi_{X}$ is $z$-closed. See $\S 5$ for a discussion of this problem, and for remarks concerning the presumably simpler question of when $\pi_{X}$ is closed. The latter, too, has not been completely answered although many results have been obtained (e.g., $[\mathrm{HM}]$, more extensively $[\mathrm{N}],[\mathrm{FF}]$ and the references given there; here, 3.4 and $\$ 5$.)

ACKNOWLEDGements. I would like to thank S. G. Mrówka for his many substantial contributions to this research. The work was begun in collaboration with Mrówka, and some of the results were reported in [HM(a)]. Those results which we obtained together and which appear here are so indicated.

I am indebted to W. W. Comfort and N. Noble for many discussions concerning this work and their papers [CN] and [N].

Received by the editors February 14, 1968. 
This research has been partially suported by the National Science Foundation under grants GP 5793 and GP 7455 at the University of Rochester.

2. Preliminaries. We first define some of the terms used in 1.2.

Let $n$ be a cardinal number. A space is $n$-discrete $\left[I_{1}\right.$, p. 135] if each intersection of $n$ or fewer open sets is open. (It seems that these spaces were studied first by Sikorski [S], whose terminology differs.) Thus, each space is $n$-discrete for finite $n$, and an $\boldsymbol{\aleph}_{0}$-discrete space is a $P$-space [GJ, $4 \mathrm{~J}$ ].

Let $n$ be an infinite cardinal. If in a space each locally finite family of open sets has power $<n$, the space is called pseudo- $n$-compact. (This is equivalent to the definition in $\left[I_{1}\right.$, p. 135].) Thus, pseudo- $\aleph_{0}$-compact=pseudocompact [G].

The following result of Noble's tells us, roughly, where to look for $z$-closed projections.

$2.1[\mathrm{~N}]$. If $\pi_{X}$ is $z$-closed, then for each infinite cardinal $n$, either $Y$ is pseudo- $n$ compact or $X$ is $n$-discrete.

(2.1 for $n=\boldsymbol{\aleph}_{0}$ was observed earlier in [CN, 2.1] and [HM (b)].) The converse of 2.1 is (very) false. There is pseudo-compact $Y$ and $\boldsymbol{\aleph}_{0}$-discrete $X$ with $\pi_{X}$ not $z$-closed ([CN, 4.6] and [HM (b)]).

So the sequel can be described briefly: we obtain some sets of conditions sufficient that $\pi_{X}$ be $z$-closed, which sets are minimal, in some sense; the conditions will be stronger than those in 2.1, and weaker than those in 1.2.

3. From the example following 2.1, it follows that pseudocompactness of $Y$ does not imply that $\pi_{X}$ is $z$-closed. But compactness of $Y$ does, of course. The following definition is involved in a generalization of this fact.

For $n$ an infinite cardinal, a space is weakly-n-compact if each open cover has a subfamily of power $<n$ with dense union. This notion was introduced by Frolík $\left[\mathrm{F}_{1}\right]$ under a different name.

(For example, weakly- $\boldsymbol{\aleph}_{0}$-compact $=$ compact; separable, and Lindelöf, spaces are weakly- $\boldsymbol{\aleph}_{1}$-compact. In general, a weakly- $n$-compact space is pseudo- $n$ compact; and for paracompact spaces, both these notions coincide with: each open cover has a subcover of power $<n$.)

3.1 THEOREM [HM (b)]. If $Y$ is weakly-n-compact, then whenever $X$ is $m$-discrete for each $m<n, \pi_{X}$ is $z$-closed.

Proof. If $n=\boldsymbol{\aleph}_{0}, Y$ is compact and the conclusion follows. So we suppose $n>\boldsymbol{\aleph}_{0}$, and that the hypotheses above are satisfied.

Let $Z$ be the zero-set of $f$, and let $x_{0} \notin \pi_{X}[Z]$. Let $k$ be a positive integer. For each $y \in Y$, choose a neighborhood $U_{y}^{k} \times V_{y}^{k}$ of $\left(x_{0}, y\right)$ on which $f$ varies less than $1 / k$. $\left\{V_{y}^{k}: y \in Y\right\}$ has a subfamily, $\left\{V_{y_{\alpha}}^{k}\right\}$, of power $<n$, with union dense in $Y$. Then $U_{k}=\bigcap_{\alpha} U_{y_{\alpha}}^{k}$ is an open set in $X$.

Let $U=\bigcap_{k=1}^{\infty} U_{k}$. Because $n>\aleph_{0}, U$ is an open set containing $x_{0}$; and $U \cap \pi_{X}[Z]$ $=\varnothing$, as we now verify. 
Actually, for each $y \in Y$, the function of $x, f(\cdot, y)$, is constant on $U$. For, let $y \in Y$ and $x \in U$. Given $\varepsilon>0$, choose neighborhoods $U_{1} \times V$ and $U_{2} \times V$ of $(x, y)$ and $\left(x_{0}, y\right)$, respectively, with $f$ varying less than $\varepsilon / 3$ on each. Choose $k$ with $1 / k<\varepsilon / 3$, and then choose $y^{\prime} \in V \cap \bigcup_{\alpha} V_{y_{\alpha}}^{k}$. Now,

$$
\left|f(x, y)-f\left(x_{0}, y\right)\right| \leqq\left|f(x, y)-f\left(x, y^{\prime}\right)\right|+\left|f\left(x, y^{\prime}\right)-f\left(x_{0}, y^{\prime}\right)\right|+\left|f\left(x_{0}, y^{\prime}\right)-f\left(x_{0}, y\right)\right| \text {. }
$$

The first and third terms are each $<\varepsilon / 3$ by the definition of $U_{1} \times V$ and $U_{2} \times V$, respectively. The second term is $<\varepsilon / 3$ because $\left(x, y^{\prime}\right),\left(x_{0}, y^{\prime}\right)$ both lie in the same $U_{y_{\alpha}}^{k} \times V_{y_{\alpha}}^{k}$.

The next result establishes that weak-n-compactness in 3.1 is the optimal condition. (See the remark following 3.2.)

3.2 TheOREM [HM (b)]. Suppose that $Y$ has the property that, for every space $X$ which is $m$-discrete for all $m<n, \pi_{X}$ is $z$-closed.

(a) If $n$ is regular (i.e., not the sum of fewer than $n$ smaller cardinals), then $Y$ is weakly-n-compact.

(b) If $n$ is not regular, then $Y$ is weakly-n*-compact, where $n^{*}$ is the least cardinal larger than $n$.

REMARK. The converse of 3.2 (b) is included in 3.1. For, if $n$ is not regular, and $X$ is $m$-discrete for all $m<n$, then $X$ is $n$-discrete [ $\mathrm{I}_{1}, \mathrm{p}$. 134]. Thus, for such $X$, if $Y$ is weakly- $n^{*}$-compact, $\pi_{X}$ is $z$-closed by 3.1 .

Proof of 3.2. We first note that (a) implies (b). If the hypotheses in (b) are satisfied, then, from the Remark above, $\pi_{X}$ is $z$-closed for every $X$ which is $m$-discrete for all $m<n^{*}$. But $n^{*}$ is regular, so (a) applies. So we prove (a).

Suppose $n$ is regular, and $Y$ is not weakly-n-compact. Choose an open cover of $Y$ with the property that no subfamily with power $<n$ has dense union, and let $\mathscr{R}$ be the set whose elements are these subfamilies of power $<n$. Let $X=\mathscr{R} \cup\{p\}$, topologized as follows. Members of $\mathscr{R}$ are isolated; given $A \in \mathscr{R}$, the set $\{p\}$ $\cup\{B: B \supset A\}$ is a neighborhood of $p$. It is easily shown that $X$ is Hausdorff. Moreover, $X$ is normal, because given two disjoint closed sets, only one can contain $p$ so the other must be open. Because $n$ is regular, the intersection of $<n$ neighborhoods of $p$ is again a neighborhood, and $X$ is $m$-discrete for $m<n$. Now, a continuous function $f$ from $X \times Y$ to the reals is defined as follows. Given $A \in \mathscr{R}$, choose $y_{A} \notin \mathrm{cl} \cup\{U: U \in A\}$. Let $f_{A}$ be a continuous function on $Y$ with $f_{A}\left(y_{A}\right)=0$, and $f_{A}(y)=1$ if $y \in \operatorname{cl} \bigcup\{U: U \in A\}$. Let $f(p, y)=1$ for all $y \in Y$, and $f(A, y)$ $=f_{A}(p)$. With $Z$ the set of zeros of $f$, it is clear that $\pi_{X}[Z]=X-\{p\}$, and that this set is not closed. Finally, $f$ is continuous. This is automatic at each $(A, y)$. Given $(p, y)$, pick $A \in \mathscr{R}$ with $y$ lying in a member $U$ of $A$. On $(\{p\} \cup\{B: B \supset A\}) \times U, f$ has value 1 , so that $f$ is continuous at $(p, y)$. The proof is complete.

4. In this section we establish a result which stands in good analogy with the sufficiency in 1.2(b). The result generalizes the sufficiency part of the following theorem of Tamano. 
$4.1\left[\mathrm{~T}_{1}\right]$. Let $X$ and $Y$ be pseudocompact spaces. $\pi_{X}$ is $z$-closed iff $X \times Y$ is pseudocompact.

Tamano's proof of the sufficiency uses the Glicksberg theorem, that $\beta(X \times Y)$ $=\beta X \times \beta Y$ if $X \times Y$ is pseudocompact [G]. A direct proof can be fashioned from an argument of Frolík [ $\mathrm{F}_{2}$, proof of 1.3]. The proof below generalizes this method.

4.2 THEOREM. If $X \times Y$ is pseudo- $n$-compact, and $X$ is $m$-discrete for each $m<n$, then $\pi_{X}$ is $z$-closed.

Proof. Let the hypotheses be satisfied. Let $Z$ be the zero-set (in $X \times Y$ ) of $f$. Suppose $x \notin \pi_{X}[Z]$. For $k$ a positive integer, let $Y_{k}=\operatorname{cl}\{y:|f(x, y)|>1 / k\}$. Then, $Z_{k}=Z \cap\left(X \times Y_{k}\right)$ is a zero-set in $X \times Y_{k}, Z=\bigcup_{k=1}^{\infty} Z_{k}$, and $\pi_{X}[Z]=\bigcup_{k=1}^{\infty} \pi_{X}\left[Z_{k}\right]$. We shall show that, for each $k, x \notin \mathrm{cl} \pi_{X}\left[Z_{k}\right]$. If $n>\aleph_{0}$, it follows that $\mathrm{cl} \bigcup_{k=1}^{\infty} \pi_{X}\left[Z_{k}\right]$ $=\bigcup_{k=1}^{\infty} \operatorname{cl} \pi_{X}\left[Z_{k}\right]$, so that $x \notin \operatorname{cl} \pi_{X}[Z]$. If $n=\aleph_{0}$, then the function on $Y, f(x, \cdot)$, is bounded away from 0 [GJ, 1G.2]; so for some $k, Y_{k}=Y$ and $x \notin \mathrm{cl} \pi_{X}[Z]$ follows.

From the definition, it follows that an open subset of a pseudo- $n$-compact space has pseudo- $n$-compact closure. So each $X \times Y_{k}$ is pseudo-n-compact. It now suffices to prove

4.3. Let $X \times Y$ be pseudo-n-compact, and let $X$ be $m$-discrete for all $m<n$. Let $Z$ be the zero-set of $f$. If, on $\{x\} \times Y$, $f$ is bounded away from 0 , then $x \notin \mathrm{cl} \pi_{X}[Z]$.

Suppose for simplicity that $f \geqq 0$. (Otherwise consider $|f|$.) Let the hypotheses in 4.3 hold, but suppose $x \in \mathrm{cl} \pi_{X}[Z]$. We generalize Frolík's inductive argument $\left[\mathrm{F}_{2}, 1.3\right]$, doing transfinite induction over the ordinals $<\omega_{n}=$ the least ordinal of power $n$.

Let $a$ be a positive real number with $f(x, y) \geqq a$ for all $y \in Y$. We shall define, for each $\alpha<\omega_{n},\left(x_{\alpha}, y_{\alpha}\right) \in Z$, and open neighborhoods $W_{\alpha}=U_{\alpha} \times V_{\alpha}, W_{\alpha}^{\prime}=U_{\alpha}^{\prime} \times V_{\alpha}$ of $\left(x_{\alpha}, y_{\alpha}\right),\left(x, y_{\alpha}\right)$, respectively, such that $U_{\alpha} \subset \bigcap\left\{U_{\beta}^{\prime}: \beta<\alpha\right\}$, and $f / W_{\alpha} \leqq a / 3, f / W_{\alpha}^{\prime}$ $\geqq 2 a / 3$.

Choose $x_{0} \in \pi_{X}[Z]$ and $y_{0}$ with $\left(x_{0}, y_{0}\right) \in Z$. Choose neighborhoods $W_{0}=U_{0} \times V_{0}$, $W_{0}^{\prime}=U_{0}^{\prime} \times V_{0}$, of $\left(x_{0}, y_{0}\right)$ and $\left(x, y_{0}\right)$ respectively, with $f \mid W_{0} \leqq a / 3$ and $f \mid W_{0}^{\prime} \geqq 2 a / 3$.

Let $\alpha<\omega_{n}$, and suppose that for each $\beta<\alpha,\left(x_{\beta}, y_{\beta}\right), W_{\beta}=U_{\beta} \times V_{\beta}$ and $W_{\beta}^{\prime}$ $=U_{\beta}^{\prime} \times V_{\beta}^{\prime}$ have been defined which have the properties mentioned. There are fewer than $n$ of the open sets $U_{\beta}^{\prime}$, so $\bigcap\left\{U_{\beta}^{\prime}: \beta<\alpha\right\}$ is open. Choose $x_{\alpha} \in \bigcap\left\{U_{\beta}^{\prime}: \beta<\alpha\right\}$ $\cap \pi_{X}[Z]$, and then $y_{\alpha}$ with $\left(x_{\alpha}, y_{\alpha}\right) \in Z$. Now choose $W_{\alpha}=U_{\alpha} \times V_{\alpha}, W_{\alpha}^{\prime}=U_{\alpha}^{\prime} \times V_{\alpha}$, neighborhoods of $\left(x_{\alpha}, y_{\alpha}\right),\left(x, y_{\alpha}\right)$, respectively, with $U_{\alpha} \subset \cap\left\{U_{\beta}^{\prime}: \beta<\alpha\right\}$, and $f\left|W_{\alpha} \leqq a / 3, f\right| W_{\alpha}^{\prime} \geqq 2 a / 3$. This completes the induction step.

By pseudo- $n$-compactness, the family $\left\{W_{\alpha}: \alpha<\omega_{n}\right\}$ cannot be locally finite, so there is a point $(\bar{x}, \bar{y})$ with each neighborhood meeting infinitely many $W_{\alpha}$. Evidently, this implies $f(\bar{x}, \bar{y}) \leqq a / 3$. But also, $f(x, y) \geqq 2 a / 3$, because each neighborhood of $(x, y)$ meets infinitely many $W_{\alpha}^{\prime}$ as well. For, if $U \times V$ is a neighborhood of $(\bar{x}, \bar{y})$, choose a countable infinity of ordinals $\alpha_{1}<\alpha_{2}<\cdots\left(<\omega_{n}\right)$ with $W_{\alpha_{i}} \cap$ $(U \times V) \neq \varnothing$ for each $i$. Since $U \cap U_{\alpha_{i+1}} \neq \varnothing$, it follows that $U \cap U_{\alpha_{i}}^{\prime} \neq \varnothing$. Thus, $W_{\alpha_{i}}^{\prime} \cap(U \times V) \neq \varnothing$, for each $i$. 
We have a contradiction, and the proof is complete.

We will discuss extensions of 4.2 shortly. First we mention a "converse" of 4.2 , due to Noble, which generalizes the necessity in 4.1.

4.4 Theorem. Suppose $X$ is $m$-discrete for each $m<n$, but not discrete, and suppose $X$ and $Y$ are pseudo-n-compact. If $\pi_{X}$ is $z$-closed then $X \times Y$ is pseudo-ncompact.

(Actually, Noble does not quite state 4.4 , but he proves it [N, 3.4].)

The question arises of what conditions on the factors make a product pseudo- $n$ compact. For the case $n=\boldsymbol{\aleph}_{0}$, much is known. See, for example [F,$\left.\S 3\right]$, [G], [SS], and some of the references in the latter two papers. Undoubtedly, many of the results for $n=\boldsymbol{\aleph}_{0}$ can be generalized. We confine the present discussion to two simple remarks, the first of which disposes of the case omitted in 4.4, of discrete $X$.

4.5. Suppose $X$ is discrete. When is $X \times Y$ pseudo- $n$-compact? Let $|X|$ denote the power of $X$, and $p$ the least cardinal such that $Y$ is pseudo-p-compact. Then: $X \times Y$ is pseudo- $n$-compact iff $|X|<n$ and either (a) $p<n$, or (b) $p=n$ and $n$ is not the sum of fewer than $|X|$ smaller cardinals. The proof is obtained by tracing locally finite families in $X \times Y$ on the subsets $\{x\} \times Y$.

The following is obtained from 4.4 and 3.1.

4.6 COROllary. Suppose $X$ is $m$-discrete for each $m<n$, and pseudo-n-compact. If $Y$ is weakly-n-compact, then $X \times Y$ is pseudo-n-compact.

This generalizes the well-known fact that $X \times Y$ is pseudocompact if $X$ is pseudocompact and $Y$ is compact [GJ, 9.14]. (Actually, in 4.6 the discreteness hypothesis on $X$ can be omitted.)

5. Some remarks. The discussion focuses around extensions and modifications of 4.2. It is rather clear that the condition that $\pi_{X}$ be $z$-closed should have little to do with global properties of $X$. For example, with very minor alterations the Proof of 4.2 works if each point of $X$ has a neighborhood $G$ such that $G \times Y$ is pseudo- $n$ compact.

Another approach derives from a desire to assume only conditions on $X$ and $Y$, and not a priori on $X \times Y$. This leads to the question: what property of $X$ is necessary and sufficient that $X \times Y$ be pseudo- $n$-compact for each pseudo- $n$-compact $Y$ ? For $n=\boldsymbol{\aleph}_{0}$, this problem has been solved by Frolík $\left[\mathrm{F}_{2}, 3.6\right]$, and the generalization shouldn't be too difficult. But again, for just the conclusion that $\pi_{X}$ be $z$-closed, local properties of $X$ ought to suffice. We are led to the question

5.1. What property of $X$ is necessary and sufficient that for each pseudo-n-compact $Y, \pi_{X}$ is $z$-closed?

(An analogous question is answered by the results in §3.)

For $n=\aleph_{0}$, it might be possible to solve this problem by "localizing" the condition $\left[F_{2}, 3.6 .1\right]$. But the following question, which should be essentially simpler, has not been answered completely: what property of $X$ is necessary and 
sufficient that for each countably compact $Y, \pi_{X}$ is closed? This question was raised by Isiwata $\left[I_{2}\right]$. The best partial answer is due to Isiwata, and Franklin and Fleischer [FF]: it is sufficient that $X$ be a subspace of some sequential space; and the converse is not known.

Returning to 5.1 for $n=\boldsymbol{\aleph}_{0}$, it is sufficient that $X$ be sequential. ([HM (b)]; recall 4.1 and compare $\left[I_{2}\right.$, p. 142, 5 (c) ].) I doubt that it suffices that $X$ be a subspace of a sequential space. But each sequential space is a $k$-space, and that $X$ be a $k$-space is sufficient $\left[\mathrm{T}_{1}\right.$, p. 229].

Next, we indicate a procedure whereby new results can be derived from known ones of a certain type. One starts, for example, with the theorem: $\pi_{X}$ is $z$-closed if $Y$ is pseudocompact and $X$ is first-countable (from 4.1 and [I, p. 142, 5 (c)]), then, by simultaneously strengthening the hypothesis on $Y$ and weakening that on $X$, one preserves the conclusion that $\pi_{X}$ be $z$-closed. This can be done in various ways; we mention two examples (without proof).

5.2 Proposition. $\pi_{X}$ is $z$-closed if $X$ has property $*$ ( $x$ is in the closure of $A$ iff $x$ is in the closure of a countable subset of $A$ ) and $Y$ has the property: given a sequence $\left\{U_{n}\right\}$ of open sets, there is a compact set $K$ such that for each $n, K \cap U_{n} \neq \varnothing$.

5.3 Proposition [HM (b)]. $\pi_{X}$ is z-closed if in X Tukey's n-phalanxes [ $\mathrm{T}_{2}$ ] determine the topology, and $Y$ is n-pseudocompact in the sense of Kennison [K].

Finally, we make some remarks on the relation between the two questions: when is $\pi_{X} z$-closed? and when is it closed?

It seems to be the case that theorems concerning the condition that $\pi_{X}$ be $z$-closed which involve hypotheses "only on the factors $X$ and $Y$ " (e.g., hypotheses such as 1.2 (a) as opposed to 1.2 (b)) have exact analogues for the condition that $\pi_{X}$ be closed.

In particular, call a space $n$-compact if each open cover has a subcover of power $<n$. If in 3.1 and 3.2, " $z$-closed" is replaced by "closed", "weakly- $n$-compact" by " $n$-compact" and "weakly- $n$ *-compact" by " $n$ *-compact", then the resulting statements are true. The proofs are simplified versions of those above ([HM], see also [N, §2].)

Continuing in this vein, 5.3 remains true if " $z$-closed" is replaced by "closed" and " $n$-pseudocompact" is replaced by the condition that each open cover of power $\leqq n$ has a finite subcover.

There also is an analogue of 5.2, which we amplify a bit.

5.4 Proposition. The following conditions on a space $Y$ are equivalent.

(a) For any space $X$ : if $F$ is a closed subset of $X \times Y$ then $\pi_{X}[F]$ contains the closure of each countable subset.

(b) For every $X$ satisfying * of 5.2, $\pi_{X}$ is closed.

(c) $Y$ has the property: each countable subset has compact closure.

(The proof of 5.4 is not difficult, and we omit it.) 
In footnote 8 of [FF] Franklin and Fleischer raise a question which I interpret to be: what spaces $Y$ have property 5.4 (a)? They note that compact spaces do, spaces which do are countably compact, and a space which does need not be sequentially compact. They ask for an example of a sequentially compact space without property 5.4 (a). Because of 5.4 (c), a separable sequentially compact space which is not compact would be an example. M. E. Rudin has constructed such a space, in $[R]$, using the continuum hypothesis. (This was pointed out to me by A. H. Stone.)

The following is easy to prove.

5.5 Proposition. If $\pi_{X}$ is $z$-closed and $X \times Y$ is normal, then $\pi_{X}$ is closed.

I know of no theorems with conclusion " $\pi_{X}$ is closed" without hypotheses solely on the factors $X$ and $Y$, except some which follow from 5.4 and a theorem (like 4.1) with conclusion " $\pi_{X}$ is $z$-closed".

6. In [ $\mathrm{I}_{1}$, III. 39] Isbell notes that the semi-uniform product $X * Y$ of fine uniform spaces $X$ and $Y$ is fine iff $X \times Y$ is $C^{*}$-embedded in $X \times \beta Y$ (i.e., each bounded continuous real-valued function on $X \times Y$ has a continuous extension over $X \times \beta Y$ ). This, and the following, suffice to prove 1.1 .

6.1 Proposition [HM (b)]. $\pi_{X}$ is z-closed iff $X \times Y$ is $C^{*}$-embedded in $X \times \beta Y$.

Proof. Let $\pi_{X}$ be $z$-closed. By [D, X.5.3], it suffices to show that, given $f, y_{0} \in$ $\beta Y-Y, x_{0} \in X$, and $\varepsilon>0$, there is a neighborhood $G$ of $\left(x_{0}, y_{0}\right)$ in $X \times \beta Y$ such that on $(X \times Y) \cap G, f$ varies by less than $\varepsilon$. Since the function on $Y, f\left(x_{0}, \cdot\right)$, extends to $y_{0}$, there is a neighborhood $V$ of $y_{0}$ such that on $Y \cap V, f\left(x_{0}, \cdot\right)$ varies by less than $\varepsilon / 3$. The zero-set $Z=\left\{(x, y):\left|f(x, y)-f\left(x_{0}, y\right)\right| \geqq \varepsilon / 3\right\}$ has closed projection on $X$, so there is a neighborhood $U$ of $x_{0}$ missing $\pi_{X}[Z] . G=U \times V$ is the desired neighborhood of $\left(x_{0}, y_{0}\right)$.

For the converse, recall that [GJ, 6.4] $X \times Y$ is $C^{*}$-embedded in $X \times \beta Y$ iff disjoint zero-sets in $X \times Y$ have disjoint closures in $X \times \beta Y$. Suppose $\pi_{X}$ is not $z$ closed, and let $f$ be a function whose zero-set $Z$ has $x_{0} \in \operatorname{cl} \pi_{X}[Z]-\pi_{X}[Z] . g(x, y)$ $=\left|f(x, y)-f\left(x_{0}, y\right)\right|$ defines a continuous function; let $Z_{1}$ be its zero-set. Evidently, $Z \cap Z_{1}=\varnothing$. Let a bar denote closure in $X \times \beta Y$. We have $\left(\left\{x_{0}\right\} \times \beta Y\right) \cap \bar{Z} \neq \varnothing$, and $\left\{x_{0}\right\} \times \beta Y \subset \bar{Z}_{1}$. So $\bar{Z} \cap \bar{Z}_{1} \neq \varnothing$, as desired.

(Independently, Comfort and Negrepontis have shown that $X \times Y$ is $C^{*}$-embedded in $X \times \beta Y$ if $\pi_{X}$ is closed [CN, 3.1]. Their proof is the same as that above.)

\section{REFERENCES}

[CN] W. W. Comfort and S. Negrepontis, Extending continuous functions on $X \times Y$ to subsets of $\beta X \times \beta Y$, Fund. Math. 59 (1966), 1-12.

[D] J. Dugundji, Topology, Allyn and Bacon, Boston, 1966.

$\left[\mathrm{F}_{1}\right] \mathrm{Z}$. Frolík, Generalizations of compact and Lindelöf spaces, Czechoslovak Math. J. 9 (1959), 172-217. (Russian. English summary) 
A. W. HAGER

$\left[\mathrm{F}_{2}\right]-$, The topological product of two pseudocompact spaces, Czechoslovak Math. J. 10 (1960), 339-349.

[FF] I. Fleischer and S. P. Franklin, On compactness and projections, (to appear)

[G] I. Glicksberg, Stone-Čech compactifications of products, Trans. Amer. Math. Soc. 90 (1959), 369-382.

[GJ] L. Gillman and M. Jerison, Rings of continuous functions, Van Nostrand, Princeton, N. J., 1960.

[HM] A. W. Hager and S. G. Mrówka, (a) Compactness and the projection mapping from a product space, Notices Amer. Math. Soc. 12 (1965), 368 (Abstract 65T-167). (b) unpublished manuscript.

[ $\left.\mathrm{I}_{1}\right]$ J. R. Isbell, Uniform spaces, Math. Surveys No. 12, Amer. Math. Soc., Providence, R. I., 1964.

[I $\left.{ }_{2}\right]$ T. Isiwata, Normality and perfect mappings, Proc. Japan Acad 39 (1963), 95-97.

[K] J. F. Kennison, m-pseudocompactness, Trans. Amer. Math. Soc. 104 (1962), 436-442.

[M] S. G. Mrówka, Compactness and product spaces, Colloq. Math. 7 (1959), 19-22.

[N] N. Noble, Products with closed projections, Trans. Amer. Math. Soc. 141 (1969).

[R] M. E. Rudin, A technique for constructing examples, Proc. Amer. Math. Soc. 16 (1965), $1320-1323$.

[S] R. Sikorski, Remarks on some topological spaces of high power, Fund. Math. 37 (1950), 125-136.

[SS] C. T. Scarborough and A. H. Stone, Products of nearly compact spaces, Trans. Amer. Math. Soc. 124 (1966), 131-147.

$\left[\mathrm{T}_{1}\right] \mathrm{H}$. Tamano, A note on the pseudo-compactness of the product of two spaces, Mem. Coll. Sci. Univ. Kyoto Ser. A Math. 33 (1960/61), 225-230.

$\left[\mathrm{T}_{2}\right]$ J. W. Tukey, Convergence and uniformity in topology, Princeton Univ. Press, Princeton, N. J., 1940.

WeSLEYAN UNIVERSITY, Middletown, Connecticut 